\title{
Improving Evidence-Based Practice through Preregistration of Applied Research: Barriers and Recommendations
}

\author{
Thomas Rhys Evans \\ Peter Branney \\ Andrew Clements
}

Ella Hatton

\footnotetext{
The current document is the final author-submitted copy of this work which has been accepted by Accountability in Research.
} 


\title{
PREREGISTRATION IN APPLIED RESEARCH
}

\begin{abstract}
Preregistration is the practice of publicly publishing plans on central components of the research process before access to, or collection, of data. Within the context of the replication crisis, open science practices like preregistration have been pivotal in facilitating greater transparency in research. However, such practices have been applied nearly exclusively to basic academic research, with rare consideration of the relevance to applied and consultancy-based research. This is particularly problematic as such research is typically reported with very low levels of transparency and accountability despite being disseminated as influential grey literature to inform practice. Evidence-based practice is best served by an appreciation of multiple sources of quality evidence, thus the current review considers the potential of preregistration to improve both the accessibility and credibility of applied research towards more rigorous evidence-based practice. The current three-part review outlines, first, the opportunities of preregistration for applied research, and second, three barriers - practical challenges, stakeholder roles, and the suitability of preregistration. Last, this review makes four recommendations to overcome these barriers and maximise the opportunities of preregistration for academics, industry, and the structures they are held within changes to preregistration templates, new types of templates, education and training, and recognition and structural changes.
\end{abstract}

Key words: Applied Research; Preregistration; Transparency; Accountability; Open Science; Grey Literature 


\section{PREREGISTRATION IN APPLIED RESEARCH}

\section{Issues with Applied Research}

Evidence-based practice is the "conscientious, explicit and judicious use of the best available evidence from multiple sources... to increase the likelihood of a favorable outcome" (Barends et al., 2014, p. 4). Evidence-based practice recommendations are commonly trusted by practitioners who solicit them, assumed to be improving strategy by informing decisions using trustworthy and high-quality evidence (Kepes et al., 2014). However, such recommendations are only as good as the quality of evidence available to inform them (Kepes et al., 2014) and there are many fields of study where the majority of evidence has been deemed questionable and problematic for drawing effective recommendations for practice (IJzerman et al., 2020). Issues with evidence quality have been reported for many specific applied practices (e.g. Organizational Change Management: Evans, 2020), broader research fields (e.g., medicine: Kane et al., 2016) and research processes and structures as a whole (Munafò et al., 2017). Standards in research practices have been facing increasing scrutiny following the replication crisis (Anvari \& Lakens, 2019) where failed attempts to replicate well-established findings (Open Science Collaboration, 2015) caused a shift in thinking and culture to prioritise research quality and transparency (Fanelli, 2018). In response, norms for preregistration, in addition to other open science practices like sharing research materials, code and data, have substantively changed to better facilitate transparency, replicability, and accountability throughout the research cycle (Nosek \& Lindsay, 2018). Such developments have been nearly exclusively applied to traditional basic research pathways where the priority is to advance academic understanding or theory; application to other research streams has been left unquestioned and unexplored.

Much research is conducted by academics, researchers, and practitioners in applied settings to create practical knowledge, with the aim of evaluating or changing practices rather than improving theory or understanding. This research conducted in applied contexts, or applied consultancy research, is hereby referred to as applied research. Academic dissemination of such works is often limited by practical constraints (e.g. finite sample size) and is further complicated by publication bias (Haddaway \& Bayliss, 2015) where the decision to publish is disproportionately biased by the outcomes of the study rather than driven by the quality of design, thereby distorting the literature to reflect larger and more statistically significant effects (Franco et al., 2014). For example, an organizational intervention based upon a wellrespected theory may well be rejected due to the null findings being perceived to be indicative of poor quality and contradictory to the basic research evidence in favor of the theory. As such, when applied works do not get published within conventional academic journals they often become part of the grey literature evidence-base instead. Subsequently, results between the grey literature and academic literature can systematically differ and often portray a conflicting account of understanding. This is known as the grey literature bias (Song et al., 2010) and is problematic for forming a coherent basis of evidence for informing practice.

Publication of applied research as grey literature is particularly problematic as norms in applied research domains have been unaltered by the replication crisis, and the lack of barriers to publication means that there is little transparency or accountability, leading to suboptimal or variable quality evidence for informing practice (Adams et al., 2017). By its very nature, grey literature often involves fewer barriers to 


\section{PREREGISTRATION IN APPLIED RESEARCH}

dissemination and no guaranteed feedback or review. Without the strict gatekeeping of quality via academic peer-review, often considered the primary role of academic editors and reviewers (Hojat et al., 2003), researchers have greater degrees of freedom to present their work as they wish. As such, applied research published as grey literature may be more susceptible to questionable research practices including selective reporting, p-hacking, and hypothesising after results are known (HARKing; Kerr, 1998). Indeed there is evidence to suggest that research often changes dramatically when transitioning to academic publication, with changes to hypotheses, variables, and analyses commonly reported (Cairo et al., 2020) and consequences reported for subsequent effect size estimations (Schmucker et al., 2017). For instance, O'Boyle et al. (2014) found that the proportion of hypotheses supported within dissertations substantially increased upon subsequent publication, due to a number of questionable research practices including dropping hypotheses that were not statistically significant, adding hypotheses with statistically significant results, reversing the directions of hypotheses, and altering data. Furthermore, there is little incentive for transparency and sharing in the publication of grey literature to overcome such issues.

Despite such concerns, one central component of evidence-based practice is drawing evidence from a number of source types, and applied research projects published as grey literature can represent an influential source of evidence to inform practice (Paez, 2017). Applied research is important because it can negotiate the academic-practitioner divide. Applied research as grey literature frequently becomes influential for evidence-based practice due to its accessibility (Mahood et al., 2013) and readiness for application (IJzerman et al., 2020). For example, applied research can be used to demonstrate the suitability, or challenge the relevance, of basic research findings for practice (Adams et al., 2017). As such, to improve the evidence-base underpinning practice recommendations, there may be value in discussing the relevance and implementation of open science practices for applied research. As preregistration of studies is a common starting point to incorporating open-science practices into research (Standen, 2019), this is the focus of the current three-part discussion. First, we review the potential role of preregistration before outlining three barriers to this for applied research: practical challenges, stakeholder roles, and the suitability of preregistration. Last, we propose four recommendations - changes to preregistration templates, new types of templates, education and training, and recognition and structural changes - to help overcome these barriers and maximise the opportunities for applied research. See Table 1 for definitions of many terms used throughout this discussion. 


\section{PREREGISTRATION IN APPLIED RESEARCH}

Table 1

Key Definitions (provided by the FORRT glossary (Parsons et al., 2021) unless otherwise stated)

Term

Data
Management
Plan

Plan
Definition
A structured document that describes the process of data acquisition, analysis, management and storage during a research project. It also describes data ownership and how the data will be preserved and shared during and upon completion of a project. Data management templates also provide guidance on how to make research data FAIR and where possible, openly available.

Evidence- $\quad$ Conscientious, explicit and judicious use of the best available evidence from
based Practice multiple sources... to increase the likelihood of a favorable outcome (Barends et al., 2014, p. 4).

Grey Occurs when the results reported in journal articles are systematically

Literature Bias different from those presented in reports, working papers, dissertations or conference abstracts (Song et al., 2010, p. 3).

HARKing HARKing (or Hypothesising After Results are Known) is "presenting a post hoc hypothesis (i.e., one based on or informed by one's results) in a research report as if it was, in fact, a priori" (Kerr, 1998, p. 196). For example, performing subgroup analyses, finding an effect in one subgroup, and writing the introduction with a 'hypothesis' that matches these results.

p-hacking Exploiting techniques that may artificially increase the likelihood of obtaining a statistically significant result by meeting the standard statistical significance criterion (typically $\alpha=.05$ ). For example, performing multiple analyses and reporting only those at $p<.05$, selectively removing data until $p<.05$, selecting variables for use in analyses based on whether those parameters are statistically significant.

Power and Statistical power is the long-run probability that a statistical test correctly Power Analysis rejects the null hypothesis if the alternative hypothesis is true. It ranges from 0 to 1 , but is often expressed as a percentage. Power can be estimated using the significance criterion (alpha), effect size, and sample size used for a specific analysis technique. There are two main applications of statistical power. A priori power where the researcher asks the question "given an effect size, how many participants would I need for X\% power?". Sensitivity power asks the question "given a known sample size, what effect size could I detect with $\mathrm{X} \%$ power?

Preregistration The practice of publishing the plan for a study, including research questions/hypotheses, research design, data analysis before the data has been collected or examined. It is also possible to preregister secondary data analyses. A preregistration document is time-stamped and typically registered with an independent party (e.g., a repository) so that it can be publicly shared with others (possibly after an embargo period). Preregistration provides a transparent documentation of what was planned at a certain time point, and allows third parties to assess what changes may have occurred afterwards. 
The more detailed a preregistration is, the better third parties can assess these changes and with that the validity of the performed analyses.

Preregistration aims to clearly distinguish confirmatory from exploratory research.

Publication
Bias and the
File Drawer
Effect

Occurs whenever the research that appears in the published literature is systematically unrepresentative of the population of completed studies. Simply put, when the research that is readily available differs in its results from the results of all the research that has been done in an area, readers and reviewers of that research are in danger of drawing the wrong conclusion about what that body of research shows (Rothstein et al., 2005, p. 1).

$\begin{array}{ll}\text { Questionable } & \text { A range of activities that intentionally or unintentionally distort data in favour } \\ \text { Research } & \text { of a researcher's own hypotheses - or omissions in reporting such practices - } \\ \text { Practices } & \text { including; selective inclusion of data, hypothesising after the results are } \\ & \text { known (HARKing), and p-hacking. }\end{array}$

\begin{tabular}{ll}
\hline Registered & $\begin{array}{l}\text { A scientific publishing format that includes an initial round of peer review of } \\
\text { the background and methods (study design, measurement, and analysis plan); } \\
\text { sufficiently high quality manuscripts are accepted for in-principle acceptance } \\
\text { (IPA) at this stage. Typically, this stage } 1 \text { review occurs before data collection, } \\
\text { however secondary data analyses are possible in this publishing format. } \\
\text { Following data analyses and write up of results and discussion sections, the } \\
\text { stage } 2 \text { review assesses whether authors sufficiently followed their study plan } \\
\text { and reported deviations from it (and remains indifferent to the results). This } \\
\text { shifts the focus of the review to the study's proposed research question and } \\
\text { methodology and away from the perceived interest in the study's results }\end{array}$ \\
\hline Replication & $\begin{array}{l}\text { The finding, and related shift in academic culture and thinking, that a large } \\
\text { proportion of scientific studies published across disciplines do not replicate } \\
\text { (e.g. Open Science Collaboration, 2015). This is considered to be due to a lack } \\
\text { of quality and integrity of research and publication practices, such as } \\
\text { publication bias, QRPs and a lack of transparency, leading to an inflated rate } \\
\text { of false positive results. Others have described this process as a 'credibility } \\
\text { revolution' towards improving these practices. }\end{array}$ \\
\hline
\end{tabular}

Open Science An umbrella term reflecting the idea that scientific knowledge of all kinds, where appropriate, should be openly accessible, transparent, rigorous, reproducible, replicable, accumulative, and inclusive, all which are considered fundamental features of the scientific endeavour. Open science consists of principles and behaviors that promote transparent, credible, reproducible, and accessible science. Open science has six major aspects: open data, open methodology, open source, open access, open peer review, and open educational resources.

\footnotetext{
Transparency Having one's actions open and accessible for external evaluation. Transparency pertains to researchers being honest about theoretical, methodological, and analytical decisions made throughout the research cycle. Transparency can be usefully differentiated into "scientifically relevant transparency" and "socially relevant transparency". While the former has been the focus of early Open Science discourses, the latter is needed to provide scientific information in ways that are relevant to decision makers and members of the public.
} 


\section{PREREGISTRATION IN APPLIED RESEARCH}

\section{Opportunities for Preregistration}

Preregistration is the practice of publicly (typically immediately but occasionally following an embargo period) publishing plans on central components of the research process (e.g. hypotheses, methods, analysis) before data collection begins. Preregistration is typically endorsed based upon claims for a range of benefits for transparency and credibility (Rubin, 2020), including differentiating between exploratory and confirmatory research which may evoke different levels of confidence, minimising questionable research practices like HARKing, and publication bias (Nosek et al., 2019). Preregistration initiatives like Registered Reports (Chambers, 2013), where preregistered plans are peer-reviewed before data collection and receive in-principle acceptance where subsequent publication is virtually guaranteed regardless of the eventual results, are of great value to basic research (Scheel et al., 2021; Soderberg et al., 2021). Indeed developments in preregistration have been well-adopted by researchers in medicine and neuro, cognitive and social psychological fields, but less so in applied areas such as occupational, forensic and educational psychology (e.g. Van't Veer \& Giner-Sorolla, 2016), with clinical trials as an unique exception. Furthermore, there is a deficit for applications which are not intended for this traditional academic publishing model. Proponents have argued that preregistration can be of benefit to all types of research (Mellor \& Nosek, 2018) but very little has been done outside mainstream basic academic research aimed at improving academic theory and understanding rather than practice. Based upon the concerns around transparency and accountability of applied research discussed above, preregistration could be a fruitful strategy to improve the credibility and transparency of evidence outside of academic journal publications.

In representing a transparent and often public account of the research process, preregistration of applied research would be of significant value to minimise researcher degrees of freedom. Analogous to the way in which preregistration is applied to basic academic research, quality preregistration could limit opportunities to change decisions (without transparent reporting and justifications), selectively report findings of interest, and HARK (Nosek et al., 2019). For example, mandated preregistration of clinical trial outcomes contributed to a substantive increase in the likelihood of reporting null results (Kaplan \& Irvin, 2015). Providing a more comprehensive and transparent account of the research process (not just what was completed but also the process) protects researchers from pressure to report positive outcomes (Olken, 2015) and would provide a more constructive basis for extracting and synthesising quality evidence for informing practice. As such, in the absence of higher standards of transparency, preregistration can hold value for improving the credibility of the research (Rubin, 2020).

Preregistration may also be of benefit for countering the grey literature bias and file-drawer effect. For example, these concerns could be mitigated through a public record of the preregistration regardless of whether the final project was published or even completed. For applied research, there are often proposals that were never fully implemented, data was not analysed or analyses were not reported (e.g. because an organizational priority took resources away from the study or a stakeholder blocked progress, etc.) or where project write-ups were not externally disseminated. Preregistration might increase accountability of the researchers to close the loop and publicly report their plans and findings more widely 


\section{PREREGISTRATION IN APPLIED RESEARCH}

to feed back into the evidence base. Even partial records would be of benefit to log activity and would provide opportunities for work to build upon a more complete view of what has been conducted, how, and to what end. Indeed we might expect that a good portion of such work would have required ethical approval and thus a minimal public record would likely involve little effort on behalf of the researcher (Nosek et al., 2018). Indeed, there are relatively few reasons why ethics submissions could not be embargoed then publicly archived in a similar manner to preregistrations.

Beyond these, a number of additional potential advantages could be anticipated. For example, a public preregistration of applied research would be more accessible for incorporation into systematic reviews (Mahood et al., 2014) and could open up opportunities for collaboration and to build more diverse and experienced teams. There might also be the opportunity to improve research quality by securing feedback before data collection. Finally, adoption of preregistration may encourage wider norm changes and adoption of other open practices. For example, by preregistering a data management plan, researchers might be motivated to consider how they could share data and/or materials. Given that many applied works include proprietary measurement tools and have bespoke outcome assessments, such developments may be of benefit to resolve inconsistencies in bodies of evidence.

Whilst the majority of benefits of preregistration are typically positioned towards confirmatory quantitative research (Branney et al., 2019), the potential gains for exploratory and qualitative applied research, where subjectivity and flexibility are inherent qualities of the research, can be similar to those of quantitative designs (Haven \& van Grootel, 2019; Dirnagl, 2020). For qualitative research, preregistrations can acknowledge the paradigms and values which will inform the design and analysis, the research questions and designs can be audited for congruence, and plans can be openly available for scrutiny yet be iterative to account for changes during the process. In this regard, preregistration of applied qualitative research could similarly contribute to transparency, credibility and visibility.

\section{Barriers surrounding Applied Research Preregistration}

There are a number of unique features of applied or consultancy research which could be seen as problematic for implementing preregistration. Concerns surrounding applied research preregistration can be seen across three broad themes: practical challenges, stakeholder roles, and the suitability of preregistration. Please see Table 2 for a summary.

1. Practical: The purpose of preregistration is not just to represent a transparent account of the research conducted, but to allow reviewers, readers and users of the research to differentiate between findings which were hypothesis-driven and thus intentionally targeted (confirmatory), and those which were datadriven and found opportunistically (exploratory). To do this requires stating upfront a comprehensive plan of the research, front-loading the planning work of a research project to later detail how, why and when deviations occur. As such, preregistration could be perceived as problematic for applied research where timeframes tend to be under external control, short, and/or when practical challenges can influence the 


\section{PREREGISTRATION IN APPLIED RESEARCH}

need, study design, analysis plan or data by the time it has all been carefully logged and implemented (Nosek et al., 2018). In principle, a detailed research plan is often required by institutional ethics boards so is unlikely to represent significant additional work, however such documents are often not public, deviations are often complex to log, and they require varying levels of detail and thus offer differing levels of flexibility (Nosek et al., 2018). As such, preregistration should not be problematic where applied researchers work under the need for rigorous ethical approval, and indeed this could represent a significant portion of the work. However, the high level of detail across the research process is not the norm for ethics applications, and ethical approval is infrequently required for practitioners within commercial organizations, thus to establish the benefits expected from preregistration researchers would have to produce detailed plans that could be shared (publicly or embargoed) and updated.

Preregistration may also create additional demands for the research which may make it infeasible in practice. For example, using a power analysis to inform sample size estimates is typical within preregistrations but would be redundant where populations are small (e.g. due to a limited number of individuals working in a company or undergoing a certain intervention). There are many ways to determine and justify sample size targets, including through the acknowledgement of resource constraints (Lakens, 2021), however strategies to mitigate implications for the higher likelihood of error (e.g. by adjusting statistical analyses or changing study design) often require additional resources and expertise. Such negotiations between feasibility and rigor are common in applied research (Paine \& Delmhorst, 2020) and may not always be easy to resolve. For example, even reporting broad information about the nature of the research conducted could contain information that identifies participants or stakeholders, or which could be commercially sensitive. Such concerns around sharing sensitive information, and particularly subsequent research data, are well voiced in the open data community (Gewin, 2016), especially when applied to psychiatric or clinical fields (Walsh et al., 2018). Whilst a good preregistration is likely to include a high level of detail across the research process, this does not necessitate any given piece of information to be fully open. Embargoes, providing partial information following removal of potentially identifying combinations of sensitive detail, or other gatekeeping approaches, are all alternative strategies which can facilitate openness but require additional resources to negotiate and manage.

2. Stakeholders: By its very nature, applied research typically involves a wide range of stakeholders (Fisher, 2009) including participants, funders, managers, employees, and interest groups, representing a number of potential barriers, including the need to discuss what can be preregistered and where. It is likely that stakeholders, particularly those outside of medical fields and academia, may require additional information to appreciate what preregistration constitutes. Currently there is little of a shared language

to facilitate such discussions, extenuating concerns about what of the work could be sensitive and shareable. Many negotiations are expected to be required in this domain. For example, if brought in to design and evaluate a specific training intervention, how much detail about the content of the intervention would be permissible to share? Reporting in greater detail would facilitate transparency and support replications and statements of the value of theories adopted to inform materials. However, such materials are likely to be considered Intellectual Property and may be particularly valuable to commercial 


\section{PREREGISTRATION IN APPLIED RESEARCH}

stakeholders where it can represent advantage against competitors, leading the stakeholders to prevent or limit transparency and sharing.

Before the need to negotiate the practicality of preregistration with external parties, there is a need to acknowledge the lack of incentives. Whilst initiatives like journal badges and preregistration challenges are changing preregistration norms within academia (e.g. Kidwell et al., 2016), there are currently few direct benefits for other stakeholders to support preregistration. While academics are rewarded for publishing research, practitioners are not (Olenick et al., 2018), let alone preregistering research. Clients are unlikely to value the production of such research materials and logs. Furthermore, commercial organisations are reported to rarely prioritise or resource a high-quality evaluation of interventions (Briner \& Walshe, 2013). Instead, unexpected outcomes and post-hoc interpretations of findings are commonly reported. There are also concerns that many managers do not value research evidence as a guide to decisions (Barends et al., 2017; Rynes et al., 2018). As such, preregistration is unlikely to be demanded by clients and indeed could be perceived as an additional complication to contract, timely work completion, and project budget. Furthermore, preregistration may be considered a barrier to stakeholder flexibility and subsequent decision-making and has the capacity to threaten reputation. For example, if decisions were made late in the process, or emphases of the project were changed, this may reflect on the organisation or group making the decision and thus could be considered a reputational risk. Preregistrations are plans not prisons (DeHaven, 2017), however even transparently reported deviations could be perceived as undesirable and may contribute to resistance to preregistration.

3. Preregistration as a Method: Preregistration does not currently represent an essential business activity or indeed skill taught or experienced as part of research training e.g. in undergraduate or postgraduate courses (Blincoe \& Buchert, 2020), with some rare exceptions (e.g. Button et al., 2020). It is also nearly completely absent from discussions of the consultancy cycle or evidence-based practice. We can therefore anticipate issues surrounding preregistration quality, with insufficient detail and focus to explore what was intended and what deviations were made. Even in the fields where preregistration currently holds relevance and is taught, the quality and accessibility of preregistration documentation is problematic. In the applied domain of clinical trials for example, there are substantive issues with the consistency in outcomes reported between trial preregistrations and journal articles (Goldacre et al., 2019), quality of clinical trial reporting (Goldacre et al., 2018), and timely publication of results - even in the presence of a legal reporting requirement (DeVito et al., 2020). As a result, of the 10 companies which had drugs approved by the US Food and Drug Administration in 2012, only 2 had disclosed all trials and complied with legal disclosure requirements (Miller et al., 2015). Preregistration may appear easy, particularly with accessible templates (e.g. aspredicted.org), however the level of detail in the preregistration is of direct consequence to the benefits of preregistration in differentiating between confirmatory and exploratory work (Bakker et al., 2020). Poor quality preregistrations add nothing to the transparency of research and could indeed support poorer decision-making should the use of preregistration (independent of its quality) misleadingly increase confidence in the claims made. Current education, norms and practices represent meaningful and ongoing barriers to preregistration quality. 


\section{PREREGISTRATION IN APPLIED RESEARCH}

Finally, preregistration is often based upon templates, and similar to the problems with individuals' awareness and understanding highlighted above, these require ongoing refinement. Current preregistration templates are not fully fit for purpose in being applied to applied research. For example, in the most popular templates there are no sections dedicated to discussing the role of the stakeholders or acknowledging conflict of interests (e.g. Chivers, 2019). By shifting the focus of accountability from the individual to the document, the preregistration is likely to only be as good as the template adopted (Bakker et al., 2020). This leaves scope for suboptimal practices, omissions, and obfuscation further compromising the quality and thus advantages of preregistration.

\section{Recommendations}

Given the extent of barriers to preregistration of applied research highlighted, meaningful change is necessary before norms are likely to change. Should preregistration hold potential value for some applied research, the following four recommendations are proposed to overcome these barriers and maximise the opportunities for preregistration for academics, industry, and the structures they are held within. See Table 2 for a summary of these barriers and recommendations.

1. Preregistration Templates: There are a wide number of preregistration templates, considering broad fields (e.g. social psychology; Van't Veer \& Giner-Sorolla, 2016), different types of data (e.g. secondary data; Van den Akker et al., 2019) and different analysis strategies (e.g.coordinated data analysis; Willroth et al., 2021). See osf.io/zab38 for a list of common templates. Across such templates there are many shared features, and we anticipate that ethical applications would facilitate completion of most standard preregistration questions (e.g. the OSF and aspredicted.org). However, the current discussion has highlighted nuances for preregistration in applied practice where more relevant and accessible templates could encourage wider use and more comprehensive detail capture to facilitate more accountable practice. Adding such additional requirements to existing templates under 'Other' sections, or developing unique templates for preregistration of applied research, are similarly possible. In addition to addressing questions from the templates available they deem most relevant to the project, we encourage researchers to consider the following questions, as a minimum, until ethics boards require detailed disclosure and facilitate public/embargoed dissemination:

1. What is the date, time and stage in the research process during the current preregistration? (e.g. study design, pre-data collection, etc.)

2. What has been agreed with any stakeholders/collaborators as to what can be disclosed? This can include the extent of confidentiality agreements, meta-data such as the Organisation/Group names (e.g. agreeing on "Modern University in the UK" rather than disclosing the stakeholder group name), extent of expected sharing of other materials and data (e.g. what can be shared on the intervention content developed, data collected, etc.) and extent of sharing possible for decision-making behind future deviations from the preregistration. 


\section{PREREGISTRATION IN APPLIED RESEARCH}

3. On what basis was the work commissioned and are there any Conflicts of Interest or personal biases to declare in relation to the commission or completion of the research? (E.g. use of personal networks to secure contracts, consultancy fees, etc.)

4. Where do you anticipate deviations from this preregistration are likely? This should include discussions of certainty on each stage of the research design/process.

5. In as much detail as is permissible, what is your specific population of interest and to what extent are findings likely to generalise beyond this? (E.g. The population of interest are individuals working in the HR department of a Modern University in the UK however findings are expected to generalise across most UK HR universities employees due to the broad nature of inquiry).

6. Beyond the hypotheses/research questions stated, do any stakeholders have any different/other predictions or expectations that may contribute to bias?

2. Different Types of Preregistration: There are many different types of preregistration with varying levels of detail and fixed stages required e.g. Registered Reports (Chambers, 2013). Given the greater likelihood of substantive changes, external influences and/or time pressures within applied research, a less static preregistration format may be more feasible and encourage greater transparency and more systematic logging of iterative changes. Whilst a new preregistration form could be written for each change, a more flexible and incremental preregistration structure is proposed. The following questions are recommended as a minimum to build upon previous preregistration attempts as a transparency log.

1. What is the date, time and stage in the research process during the current preregistration? (e.g. study design, pre-data collection, etc.)

2. What is the exact nature of the change? (e.g. changing recruitment strategy from notices on bulletin boards to handing out leaflets during meetings)

3. What is the date and time when the change is/was implemented? (e.g. 9am UTC, 23rd Jan 2022)

4. Who instigated the change and on what basis was this agreed? (e.g. The Research Lead was concerned about poor recruitment levels relative to the study timeframe, and the strategy was agreed with the organisations' lead on the project, the Researcher's ethics board, and the Managers leading meetings where recruitment was encouraged)

As discussed above, it can be anticipated that changes may be sensitive and thus the transparent log of changes recommended here could be problematic in practice. Agreeing a strategy for presenting such changes in advance, as highlighted by the preregistration template above, may go some way to negotiating or preventing potential issues. Such information is valuable to report as transparently as possible, however we anticipate this area may require more substantive change should preregistration norms change e.g. preregistration templates remain editable up to the point of data collection, where changes made only after the start of data collection are reported publicly via transparency logs, etc.

3. Better Education: High-quality preregistrations are difficult and require substantive structure, support and understanding to secure the expected benefits (Bakker et al., 2020). Teaching such principles is typically not compulsory by major accrediting bodies, although some are beginning to facilitate such 


\section{PREREGISTRATION IN APPLIED RESEARCH}

developments e.g. the British Psychological Society (Branney et al., 2019). As such, introducing preregistration into research or skills-based curricula represents a substantive pedagogical opportunity to increase future engagement (Pownall, 2020). Personal experience with preregistration would be particularly beneficial and seems feasible for many scientific fields through empirical project work in undergraduate, postgraduate and doctoral-level studies. Preregistration could be easily built in, as indeed many of the processes involved are closely aligned to those expected from ethics applications and project planning (Pownall, 2020). Whilst preregistration may be perceived as time-consuming by students who are completing their research within relatively short and constrained timeframes, this context does not necessitate the need for external peer-review (as required for Registered Reports) and would represent a realistic experience of the pressures of preregistration within applied research domains. Equipping students with an understanding of preregistration could thereby support their development as advocates of the practice in academic and industry roles and the confidence established from such experience may be of strategic benefit to the educational institutions should they lead to research outputs and external dissemination. Completion of preregistration templates may also be useful for training and induction for practitioners, providing an opportunity to discuss company hierarchy and norms, observe and evaluate established practices, and negotiate expectations for outcomes.

Norms around open science practices are changing dramatically, and pedagogical attempts like the Framework for Open and Reproducible Research Training (FORRT) project look to improve future adoption (FORRT, 2019). However, these initiatives do not account for those attempting to overcome current barriers to preregistration of applied research. Open practices like preregistration have been predominantly championed by Early Career Researchers, where greater resistance has been evidenced by established academics who often have an entrenched workflow and for whom the current system has been fortunate (Abele-Brehm et al., 2019; Toribio-Flórez et al., 2021). Awareness, understanding, and a convincing case for the need for change is therefore needed for a wide range of stakeholders to secure buy-in. For the reasons highlighted above, there is likely to be much hesitancy and resistance to preregistration with concerns often surrounding the perceived increase in cost for potential additional work and the potential for problems with inflexibility and need for justification of changes. Clear education on the role and value of preregistration is necessary, catered to each stakeholder type, for which we hope the current manuscript will encourage development in these areas.

4. Recognition and Structural Change: Finally, changes to research practices should occur in context of an evolving environment that recognises and rewards efforts towards improvement of evidence dissemination and thus practice (Nosek et al., 2018). There are many intervention types from which norms surrounding open behaviours like preregistration can change, including education, modelling and coercion (Norris \& O'Connor, 2019). Given the sensitive nature of many types of applied research, mandatory preregistration policies (or negative implications for a lack of preregistration) are likely to be ineffective and problematic. Initiatives like the San Francisco Declaration on Research Assessment (DORA; Cagan, 2013) and Transparency and Openness Promotion Guidelines (TOP; Nosek et al., 2015) are changing norms around assessment of researchers with respect to impact factors and many Universities have begun requesting open science practices explicitly within recruitment materials (Lazarević \& Žeželj, 2018; see https://osf.io/4xnc7/). Preregistration of applied research would therefore be an excellent way 


\section{PREREGISTRATION IN APPLIED RESEARCH}

to evidence such skills and commitment to open practices and real-world impact in selection or promotion contexts without the emphasis on journal publications and associated metrics.

To completely reorientate research culture towards transparency and rigor, new and accessible infrastructure is needed, communities need to establish clear norms, and appropriate incentives and policies should be agreed upon to reinforce these (Mellor, 2021; Nosek, 2019). One example initiative to facilitate recognition and incentivise preregistered applied research could be through additions to the existing publication pathways. Currently there are only a few journals which explicitly encourage applied work and reflections upon applications to real-world settings e.g. Professional Psychology: Research and Practice. Introducing a new journal publishing preregistered applied research would be an opportunity to reward researchers with desired outcomes (publications, citations, external esteem) through existing pathways. The journal might be expected to have a broad remit with respect to contexts of application, a sensitive approach to negotiating what details can/cannot be published, and a community which can help refine nuanced templates such as those presented above. It could even hold editorial policies to offer inprinciple acceptance (similar to Registered Reports; Chambers, 2013) based upon the preregistration quality, and could require both academic and practitioner reviewers for each submission to ensure relevance to their respective communities. Such an initiative should be intended as short-to-medium in timeframe to generate culture change such that new recognition and rewards could be developed outside of current (problematic) assessment and incentive structures (Munafò et al., 2017). 


\section{PREREGISTRATION IN APPLIED RESEARCH}

\section{Table 2}

Barriers and Recommendations for Preregistration in Applied Research

Barriers

1. Practical issues

- Additional work required e.g. power analysis or sample size justification

- Perceived complications for timeframes and project completion

- Concerns over sharing sensitive information
Recommendations

- Adopt an accessible, flexible and incremental preregistration template to provide structure

- Use ethics applications (where required) to help minimize the work required

- Complete a transparency log as and when changes are made, accepting compromises between practicality and rigor

- Make work as open as is possible and closed as necessary by considering partial sharing, embargoes, or other gatekeeping approaches when concerns over sensitivity arise

2. Limited stakeholder awareness and engagement

- Potential lack of stakeholder awareness of preregistration

- Lack of incentives for stakeholder engagement
- Use preregistration templates as an opportunity to discuss with each stakeholder what can be shared and why that might be of value

- Take opportunities to make investments towards transparency highly visible, both internally and publicly

- Champion initiatives to drive structural and individual change towards revised norms and infrastructures which prioritise rigor and transparency

- Embrace unique routes for dissemination and publication of preregistered applied research to maximise accessibility and recognition
3. Preregistration as a method

- Current preregistration templates not being fully fit for applied research

- Preregistration quality

- Rarity of preregistration within education, and consultancy cycle or evidence-based practice discussions
- Develop templates for preregistration of applied research. The questions proposed above can be added to existing preregistration templates where necessary

- Facilitate preregistration through changes to established education and research structures such as institutional ethics boards

- Incorporate preregistration into teaching of research (e.g. research methods) and, wherever possible, provide experience of preregistration in research and skills-based curricula to increase understanding and confidence e.g. as part of obtaining ethics for dissertation research 


\section{PREREGISTRATION IN APPLIED RESEARCH}

\section{Concluding Thoughts}

When conducted in applied and consultancy research, preregistration has the potential to improve the body of evidence informing practice recommendations by minimising both researcher degrees of freedom and the file drawer effect (Nosek et al., 2019). Preregistration represents a low-cost intervention which requires little investment but which can help improve transparency, credibility and encourage collaboration. It can also be flexibly applied to meet the demands of the researcher and project, and can discourage, minimise and/or prevent many of the concerns associated with the questionable norms in basic research discussed.

The reality of implementing preregistration is perhaps a little more complex, however. Preregistration does not inherently improve research quality and in many circumstances can realistically expect to require important resources to effectively manage (Szollosi et al., 2019). We need to recognise the inherent tension in where the value of preregistration stands. The benefit mostly lies with transparency and credibility in evidence, for which the most obvious benefactor is the scientific community. Individual researchers don't have to endorse the normative Mertonian principles of science being open and of community ownership to engage with open behaviours however (Cohoon \& Howison, 2021). Publicly providing more detail and rigor in their applied projects is an opportunity to receive wider recognition for work, particularly by selection/promotion/tenure committees. As preregistration norms change, these rewards only become more certain, particularly in context of researcher assessments which increasingly prioritise real-world impact upon organisations, industries and societies. Indeed, preregistration of applied research seems likely to increase credibility and legitimacy of scientific practitioners and their communities. Towards this norm change, we should consider enacting a wider range of structural and individual intervention types (Norris \& O'Connor, 2019), and take opportunities to make investments towards transparency highly visible (Kraft-Todd \& Rand, 2021). For the organisations and industries which support preregistration, the rewards are far less certain. Wider adoption and facilitation of preregistration should help improve the body of evidence upon which organisations make decisions, and the preregistration itself could reflect a signal of trustworthiness which could be a desirable reputational gain. These are far less-concrete gains that represent long-term investments rather than easily-demonstrable outcomes, and it seems likely that until norms across research change, resistance should be expected and this cannot be considered unreasonable.

Preregistration in the current form is therefore likely to be inadequate for the quality of preregistration necessary to reap rewards for all stakeholders. In the short-term, some changes to practices, such as templates, iterative designs, and education initiatives, will be required to carry the burden until changes in practices and norms are realised. There is real potential for benefits to researchers, organisations, and the wider structures and societies within which they operate, should we manage to address the blindspot of open science practice application beyond basic research. 


\section{References}

Abele-Brehm, A. E., Gollwitzer, M., Steinberg, U., \& Schönbrodt, F. D. (2019). Attitudes toward open science and public data sharing. Social Psychology. 50(4), 252-260. https://doi.org/10.1027/1864-9335/a000384

Adams, R. J., Smart, P., \& Huff, A. S. (2017). Shades of grey: guidelines for working with the grey literature in systematic reviews for management and organizational studies. International Journal of Management Reviews, 19(4), 432-454.

Anvari, F. \& lakens, D. (2019). The replicability crisis and public trust in psychological science. Comprehensive Results in Social Psychology, 3(3), 266-286.

Bakker, M., Veldkamp, C. L., van Assen, M. A., Crompvoets, E. A., Ong, H. H., Nosek, B. A., ... \& Wicherts, J. M. (2020). Ensuring the quality and specificity of preregistrations. PLoS Biology, 18(12), e3000937.

Barends, E., Rousseau, D. M., \& Briner, R. B. (2014). Evidence-based management: The basic principles. Center for Evidence-Based Management.

Barends, E., Villanueva, J., Rousseau, D.M., Briner, R.B., Jepsen, D.M., Houghton, E., \& ten Have, S. (2017). Managerial attitudes and perceived barriers regarding evidence-based practice: An international survey. PLoS One, 12 (10), e0184594.

Branney, P., Reid, K. , Frost, N., Coan, S., Mathieson, A. \& Woolhouse, M. (2019) A context-consent meta-framework for designing open (qualitative) data studies. Qualitative Research in Psychology, 16(3), 483-502.

Briner, R. B., \& Walshe, N. D. (2013) The causes and consequences of a scientific literature we cannot trust: An evidence-based practice perspective. Industrial and Organizational Psychology, 6, 269312.

Button, K. S., Chambers, C. D., Lawrence, N., \& Munafò, M. R. (2020). Grassroots training for reproducible science: a consortium-based approach to the empirical dissertation. Psychology Learning \& Teaching, 19(1), 77-90.

Cagan, R. (2013). The San Francisco declaration on research assessment. Disease Models and Mechanisms, 6(4), 869-70.

Cairo, A. H., Green, J. D., Forsyth, D. R., Behler, A. M. C., \& Raldiris, T. L. (2020). Gray (literature) matters: Evidence of selective hypothesis reporting in social psychological research. Personality and Social Psychology Bulletin, 46(9), 1344-1362.

Chambers, C. D. (2013). Registered reports: a new publishing initiative at Cortex. Cortex, 49(3), 609-610.

Chivers, T. (2019). Does psychology have a conflict-of-interest problem?. Nature, 571(7763), $20-24$.

Cohoon, J., \& Howison, J. (2021). Norms and Open Systems in Open Science. Information \& Culture, 56(2), 115-137.

DeHaven, A. (2017). Preregistration: A Plan, Not a Prison. Retrieved from https://cos.io/blog/preregistration-plan-not-prison/

DeVito, N. J., Bacon, S., \& Goldacre, B. (2020). Compliance with legal requirement to report clinical trial results on ClinicalTrials. gov: a cohort study. The Lancet, 395(10221), 361-369. 


\section{PREREGISTRATION IN APPLIED RESEARCH}

Dirnagl, U. (2020). Preregistration of exploratory research: Learning from the golden age of discovery. PLoS Biology, 18(3), e3000690.

Evans, T. R. (2020). Improving evidence quality for organisational change management through open science. Journal of Organizational Change Management, 33(2), 367-378

Fanelli, D. (2018). Opinion: Is science really facing a reproducibility crisis, and do we need it to?. Proceedings of the National Academy of Sciences, 115(11), 2628-2631.

Fisher, M.A. (2009). Replacing "who is the client?" with a different ethical question. Professional Psychology: Research and Practice, 40 (1), 1-7

FORRT Project Team (2019). Introducing a Framework for Open and Reproducible Research Training (FORRT). https://doi.org/10.31219/osf.io/bnh7p

Franco, A., Malhotra, N., \& Simonovits, G. (2014). Publication bias in the social sciences: Unlocking the file drawer. Science, 345(6203), 1502-1505.

Gewin, V. (2016). Data sharing: An open mind on open data. Nature, 529(7584), 117-119.

Goldacre, B., DeVito, N. J., Heneghan, C., Irving, F., Bacon, S., Fleminger, J., \& Curtis, H. (2018). Compliance with requirement to report results on the EU Clinical Trials Register: cohort study and web resource. $B M J, 362$.

Goldacre, B., Drysdale, H., Dale, A., Milosevic, I., Slade, E., Hartley, P., ... \& Mahtani, K. R. (2019). COMPare: a prospective cohort study correcting and monitoring 58 misreported trials in real time. Trials, 20(1), 1-16.

Haddaway, N. R., \& Bayliss, H. R. (2015). Shades of grey: two forms of grey literature important for reviews in conservation. Biological Conservation, 191, 827-829.

Haven, T. L., \& Van Grootel, D. L. (2019). Preregistering qualitative research. Accountability in Research, 26(3), 229-244.

Hojat, M., Gonnella, J. S., \& Caelleigh, A. S. (2003). Impartial judgment by the "gatekeepers" of science: fallibility and accountability in the peer review process. Advances in Health Sciences Education, 8(1), 75-96.

IJzerman, H., Lewis, N. A., Przybylski, A. K., Weinstein, N., DeBruine, L., Ritchie, S. J., ... \& Anvari, F. (2020). Use caution when applying behavioural science to policy. Nature Human Behaviour, 4(11), 1092-1094.

Kane, R. L., Butler, M., \& Ng, W. (2016). Examining the quality of evidence to support the effectiveness of interventions: an analysis of systematic reviews. BMJ open, 6(5), e011051.

Kaplan, R. M., \& Irvin, V. L. (2015). Likelihood of null effects of large NHLBI clinical trials has increased over time. PloS one, 10(8), e0132382.

Kepes, S., Bennett, A. A., \& McDaniel, M. A. (2014). Evidence-based management and the trustworthiness of our cumulative scientific knowledge: Implications for teaching, research, and practice. Academy of Management Learning \& Education, 13(3), 446- 466.

Kerr, N. L. (1998). HARKing: Hypothesizing after the results are known. Personality and social psychology review, 2(3), 196-217.

Kidwell, M. C., Lazarević, L. B., Baranski, E., Hardwicke, T. E., Piechowski, S., Falkenberg, L. S., ... \& Nosek, B. A. (2016). Badges to acknowledge open practices: A simple, low-cost, effective method for increasing transparency. PLoS Biology, 14(5), e1002456. 


\section{PREREGISTRATION IN APPLIED RESEARCH}

Kraft-Todd, G. T., \& Rand, D. G. (2021). Practice what you preach: Credibility-enhancing displays and the growth of open science. Organizational Behavior and Human Decision Processes, 164, 1-10.

Lakens, D. (2021). Sample Size Justification. https://doi.org/10.31234/osf.io/9d3yf

Lazarević, B., \& Žeželj, I. (2018). How open science norms improve scientific practices. University of Belgrade-School of Electrical Engineering and Academic Mind, Belgrade, Serbia, 13-15.

Mahood, Q., Van Eerd, D., \& Irvin, E. (2014). Searching for grey literature for systematic reviews: challenges and benefits. Research Synthesis Methods, 5(3), 221-234.

Mellor, D. T., \& Nosek, B. A. (2018). Easy preregistration will benefit any research. Nature Human Behaviour, 2(2), 98-98.

Mellor, D. (2021). Improving norms in research culture to incentivize transparency and rigor. Educational Psychologist, 56(2), 122-131.

Miller, J. E., Korn, D., \& Ross, J. S. (2015). Clinical trial registration, reporting, publication and FDAAA compliance: a cross-sectional analysis and ranking of new drugs approved by the FDA in 2012. BMJ open, 5(11), e009758.

Munafò, M. R., Nosek, B. A., Bishop, D. V., Button, K. S., Chambers, C. D., Du Sert, N. P., ... \& loannidis, J. P. (2017). A manifesto for reproducible science. Nature Human Behaviour, 1(1), 1-9.

Norris, E., \& O'Connor, D. B. (2019). Science as behaviour: Using a behaviour change approach to increase uptake of Open Science. Psychology \& Health, 34(12), 1397-1406

Nosek, B. (2019). Strategy for Culture Change. Strategy for Culture Change. https://cos.io/blog/strategyfor-culture-change/

Nosek, B. A., \& Lindsay, D. S. (2018). Preregistration becoming the norm in psychological science. APS Observer, 31(3), 1.

Nosek, B. A., Alter, G., Banks, G. C., Borsboom, D., Bowman, S. D., Breckler, S. J., ... \& Yarkoni, T. (2015). Promoting an open research culture. Science, 348(6242), 1422-1425.

Nosek, B. A., Ebersole, C. R., DeHaven, A. C., \& Mellor, D. T. (2018). The preregistration revolution. Proceedings of the National Academy of Sciences, 115(11), 2600-2606.

Nosek, B. A., Beck, E. D., Campbell, L., Flake, J. K., Hardwicke, T. E., Mellor, D. T., ... \& Vazire, S. (2019). Preregistration is hard, and worthwhile. Trends in Cognitive Sciences, 23(10), 815-818.

O’Boyle, E. H., Banks, G. C., \& Gonzalez-Mulé. (2014). The chrysalis effect: How usly initial results metamorphosize into beautiful articles. Journal of Management, 43(2), 376-399. https://doi.org/10.1177/0149206314527133

Olenick, J. Walker, R., Bradburn, J., \& DeShon, R. (2018) A systems view of the scientist-practitioner gap, Industrial and Organizational Psychology, 11 (2), 220-266

Olken, B. A. (2015). Promises and perils of pre-analysis plans. Journal of Economic Perspectives, 29(3), 61-80.

Open Science Collaboration. (2015). Estimating the reproducibility of psychological science. Science, 349(6251).

Paez, A. (2017). Gray literature: An important resource in systematic reviews. Journal of Evidence-Based Medicine, 10(3), 233-240.

Paine, J. W., \& Delmhorst, F. (2020). A Balance of Rigor and Relevance: Engaged Scholarship in Organizational Change. The Journal of Applied Behavioral Science, 56(4), 437-461. 
Parsons, S., Azevedo, F., Elsherif, M., Guay, S., Shahim, O., Govaart, G.,... \& Aczel, B. (2021). A Community-Sourced Glossary of Open Scholarship Terms. Preprint. Glossary is available here: https://forrt.org/glossary/

Pownall, M. (2020). Pre-Registration in the Undergraduate Dissertation: A Critical Discussion. Psychology Teaching Review, 26(1), 71-76.

Rothstein, H. R., Sutton, A. J., \& Borenstein, M. (Eds.). (2006). Publication bias in meta-analysis: Prevention, assessment and adjustments. John Wiley \& Sons.

Rubin, M. (2020). Does preregistration improve the credibility of research findings?. The Quantitative Methods for Psychology. 16(3), 376-390.

Rynes, S. L., Colbert, A.E., \& O'Boyle, E. H. (2018) When the “best available evidence” doesn't win: How doubts about science and scientists threaten the future of evidence-based management. Journal of Management, 44 (8), 2995-3010.

Scheel, A. M., Schijen, M. R., \& Lakens, D. (2021). An excess of positive results: Comparing the standard Psychology literature with Registered Reports. Advances in Methods and Practices in Psychological Science, 4(2), 25152459211007467.

Schmucker, C. M., Blümle, A., Schell, L. K., Schwarzer, G., Oeller, P., Cabrera, L., ... \& OPEN consortium. (2017). Systematic review finds that study data not published in full text articles have unclear impact on meta-analyses results in medical research. PloS one, 12(4), e0176210.

Soderberg, C. K., Errington, T. M., Schiavone, S. R., Bottesini, J., Thorn, F. S., Vazire, S., ... \& Nosek, B. A. (2021). Initial evidence of research quality of registered reports compared with the standard publishing model. Nature Human Behaviour, 1-8.

Song, F., Parekh, S., Hooper, L., Loke, Y. K., Ryder, J., Sutton, A. J., ... \& Harvey, I. (2010). Dissemination and publication of research findings: an updated review of related biases. Health Technology Assessment, 14(8), 1-193.

Standen, E. (2019). Open science, pre-registration and striving for better research practices, Psychological Science Agenda. Available from: https://www.apa.org/science/about/psa/2019/11/better-research-practices

Szollosi, A., Kellen, D., Navarro, D., Shiffrin, R., van Rooij, I., Van Zandt, T., \& Donkin, C. (2019). Is preregistration worthwhile?. Trends in Cognitive Sciences, 24(2), 94-95.

Toribio-Flórez, D., Anneser, L., deOliveira-Lopes, F. N., Pallandt, M., Tunn, I., \& Windel, H. (2021). Where do early career researchers stand on open science practices? A survey within the Max Planck Society. Frontiers in Research Metrics and Analytics, 5, 17.

Van den Akker, O., Weston, S. J., Campbell, L., Chopik, W. J., Damian, R. I., Davis-Kean, P., ... \& Bakker, M. (2019). Preregistration of secondary data analysis: A template and tutorial. Doi:10.31234/osf.io/hvfmr

van't Veer, A. E., \& Giner-Sorolla, R. (2016). Pre-registration in social psychology-A discussion and suggested template. Journal of Experimental Social Psychology, 67, 2-12.

Walsh, C. G., Xia, W., Li, M., Denny, J. C., Harris, P. A. \& Malin, B. A. (2018). Enabling open-science initiatives in clinical psychology and psychiatry without sacrificing patients' privacy: Current practices and future challenges. Advances in Methods and Practices in Psychological Science, 1(1), 104-114. 


\section{PREREGISTRATION IN APPLIED RESEARCH}

Willroth, E. C., Graham, E. K., \& Mroczek, D. K. (2021). Challenges and opportunities in preregistration of coordinated data analysis: A tutorial and template. Psychology and Aging.

https://doi.org/10.1037/pag0000611 


\section{PREREGISTRATION IN APPLIED RESEARCH}

\section{Acknowledgements}

The current manuscript was first scoped out in a session on preregistration of applied research during an United Kingdom Open Science Working Group (UKOSWG) meeting. Our thanks go to all individuals in this session who contributed to the discussions that informed the current manuscript but which chose not to be involved with the current publication. We also wish to express our gratitude to Dr Madeleine Pownall (ORCID: 0000-0002-3734-8006) and one anonymous reviewer who provided detailed and insightful comments which helped improve the content and quality of discussion.

\section{Conflict of Interest}

The authors wish to declare no conflict of interest and acknowledge no funding sources.

\section{Contribution Statement}

TRE was responsible for project administration, supervision and writing (original draft). All authors contributed through conceptualisation and writing (review \& editing). 